\title{
Reconstruction and analysis of genome-scale metabolic model of weak Crabtree positive yeast Lachancea kluyveri
}

\section{CURRENT STATUS: POSTED}

Research Square

\section{Piyush Nanda}

Indian Institute of Technology Kharagpur

Pradipta Patra

Indian Institute of Technology Kharagpur

Manali Das

Indian Institute of Technology Kharagpur

Amit Ghosh

Indian Institute of Technology Kharagpur

amitghosh@iitkgp.ac.inCorresponding Author

ORCiD: https://orcid.org/0000-0003-3514-885X

\section{DOI:}

10.21203/rs.2.16651/v1

\section{SUBJECT AREAS}

Epigenetics \& Genomics

\section{KEYWORDS}

Metabolic Flux Analysis, Non-conventional yeast, Dynamic Flux Balance Analysis, Uracil degradation, Ethyl acetate 
Abstract

Background Lachancea kluyveri , a weak Crabtree positive yeast, has been extensively studied for the unique URC pyrimidine catabolism it harbours. It produces more biomass than Saccharomyces cerevisiae due to the underlying Crabtree effect and resorts to fermentation only in oxygen limiting conditions that makes it suitable host for industrial scale protein production. Ethyl acetate, an important industrial chemical, has been demonstrated to be a major overflow metabolite during aerobic batch cultivation with a specific rate of $0.12 \mathrm{~g}$ per $\mathrm{g}$ dry weight per hour. Here, we attempted to reconstruct the metabolism of the yeast from the genome to better explain the observed phenotypes and aid further hypothesis generation.

Results We report the first genome-scale metabolic model, iPN730, using Build Fungal Model in KBase workspace. The inconsistencies in the model were manually corrected using literature and published datasets. The model comprises of 1235 reactions, 1179 metabolites and 730 genes distributed in 8 compartments. The in silico viability and the growth rates in various carbon sources show good agreement. The gene essentiality of the metabolic model also performs well in comparison to experimental data confirmed by statistical analysis. Dynamic flux balance analysis describes the growth dynamics, substrate utilization and product formation kinetics in various oxygen limited conditions. The URC pyrimidine degradation pathway incorporated into the model enables it to grow on uracil or urea as the sole nitrogen source.

Conclusion The genome-scale metabolic construction of L. kluyveri provides better understanding of metabolism, particularly that of pyrimidine metabolism and ethyl acetate production. Metabolic flux analysis using the model will enable hypotheses generation to gain deeper understanding of metabolism in weakly Crabtree positive yeast.

Full-text

Due to technical limitations, full-text HTML conversion of this manuscript could not be completed. However, the manuscript can be downloaded and accessed as a PDF.

Figures 
Fig 1

(A)

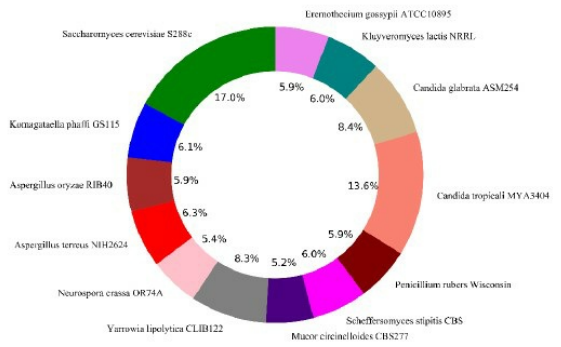

(C)

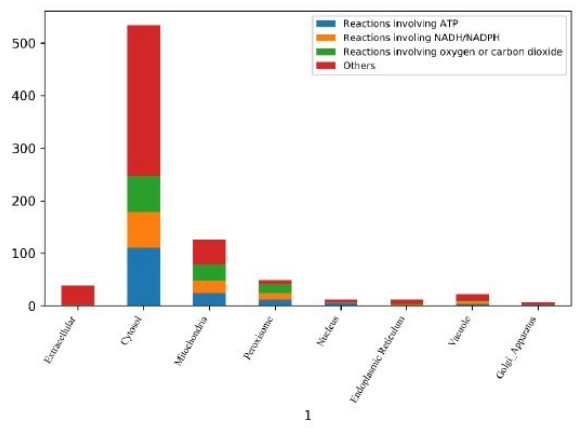

(B)

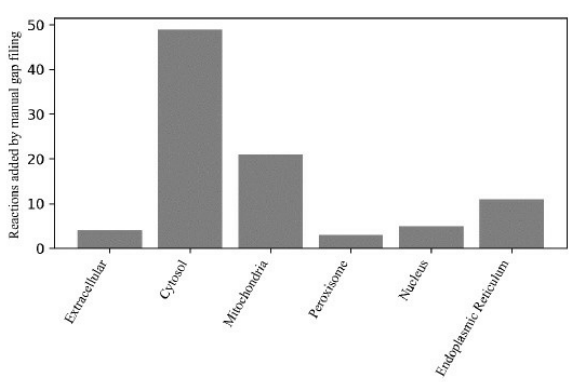

(D)

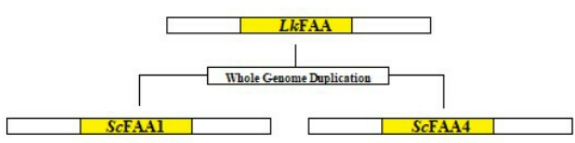

Figure 1

Genome-scale metabolic reconstruction of Lachancea kluyveri. (A) Distribution of the orthologs of Lachancea kluyveri protein coding genes in various fungi in percentage of total.

(B) The number of reactions added to the model by manual gap filling with reference to MetaCyc and KEGG contained in various compartments. (C) Irreversible reactions in the iPN730 distributed across various compartments. The colour indicates the reactions belonging specific categories involving ATP, NADH/NADPH or metabolic gases like oxygen or carbon dioxide. (D) Duplication of the prospective FAA1 (Long chain fatty acyl-CoA synthetase) in Lachancea kluyveri to FAA1 and FAA4 in Saccharomyces cerevisiae as an example of duplicate homologs. 
Fig 2

(A)

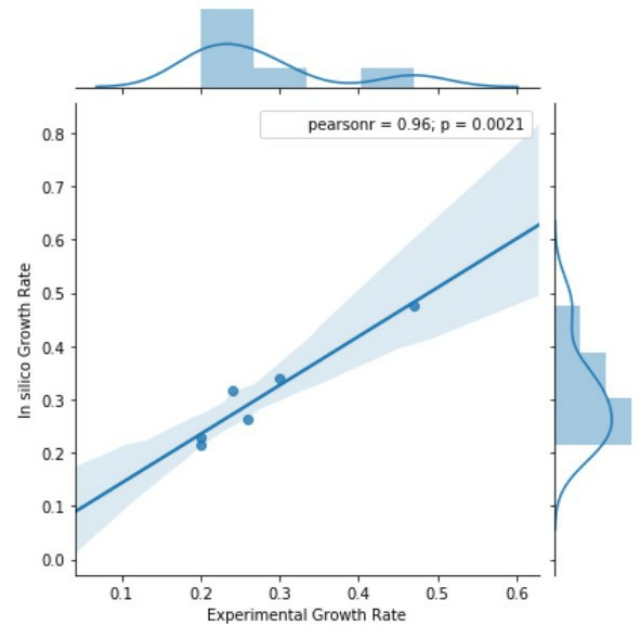

(B)

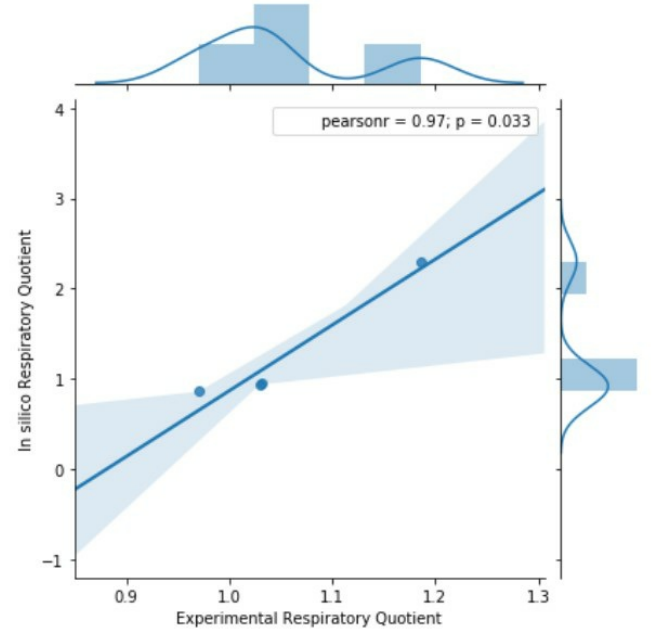

Figure 2

Computational predictions of growth characteristics show good agreement with experimental reports. (A) The correlation between the experimental growth rate observed in previous studies and the in silico growth rate from Flux Balance Analysis (FBA) of iPN730. (B) The correlation between the experimental respiratory quotient (moles of carbon dioxide released per mole of oxygen consumed and the in silico values obtained from iPN730. 
Fig 3

(A)

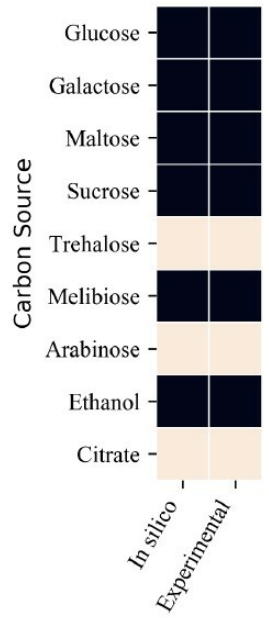

(B)

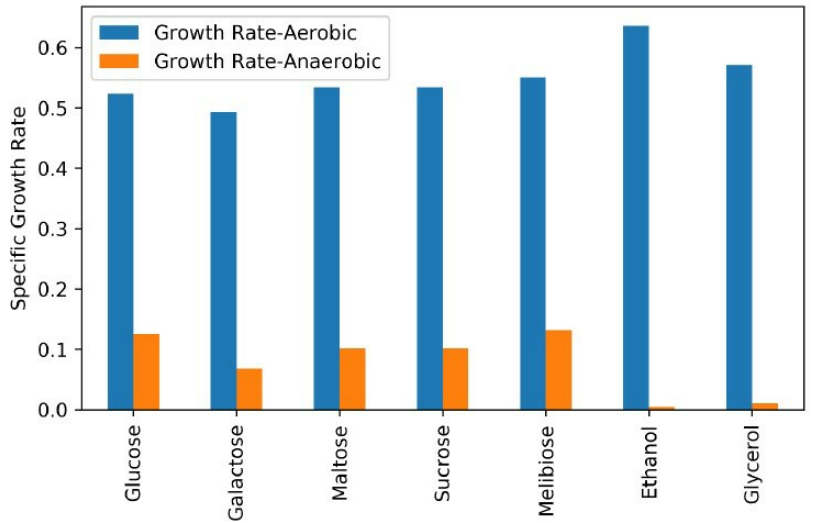

Figure 3

Viability and growth on various carbon sources in comparison to experimental reports. (A) Comparative analysis of in silico viability on multiple carbon dioxide and the reported data in MycoBank and other literatures. (B) The in silico specific growth rate (hr-1) simulated by FBA of Lachancea kluyveri on different carbon source in both aerobic and anaerobic conditions. 
Fig 4

(A)

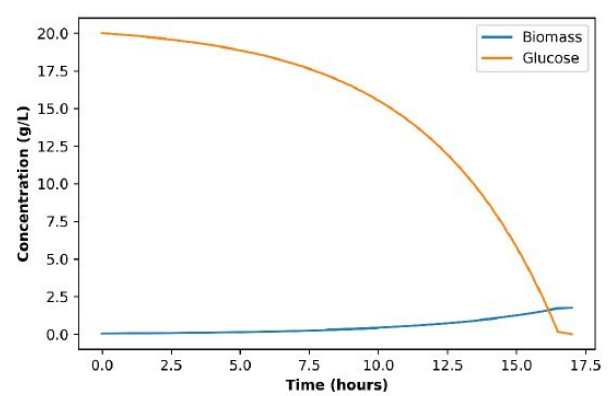

(C)

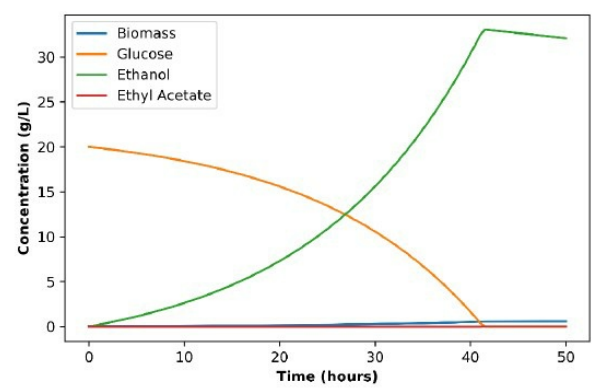

(B)

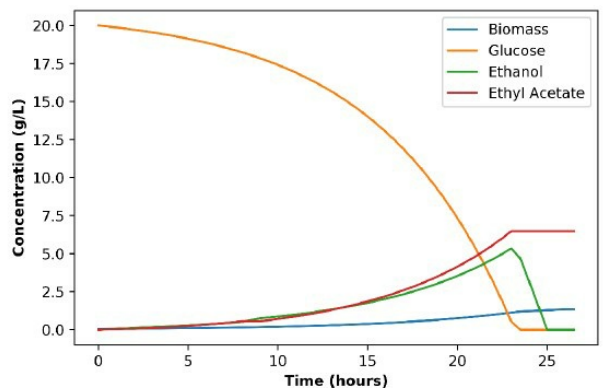

(D)

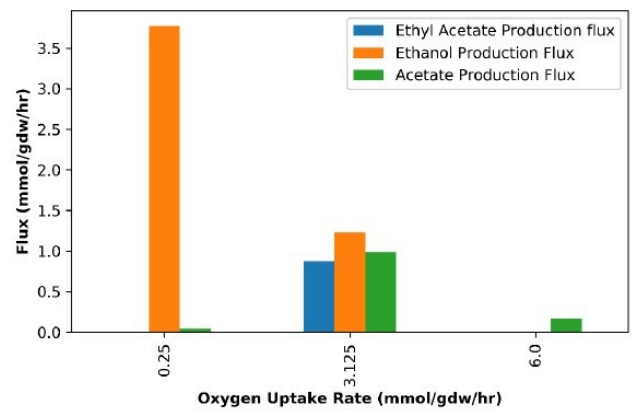

Figure 4

The dynamic flux balance analysis of iPN730 in oxygen limited conditions. DFBA on glucose as carbon source and oxygen uptake in (A) Aerobic (B) Semi-aerobic and (C) Anaerobic regime. (D) The shift from respiratory metabolism ( $6 \mathrm{mmol} g \mathrm{dw}-1 \mathrm{hr}-1$ ) to fermentative metabolism $(0.25 \mathrm{mmol} \mathrm{gdw}-1 \mathrm{hr}-1)$ shows the variation in production of acetate and ethanol which are precursor for the major overflow metabolite ethyl acetate. 
Fig 5

(A)

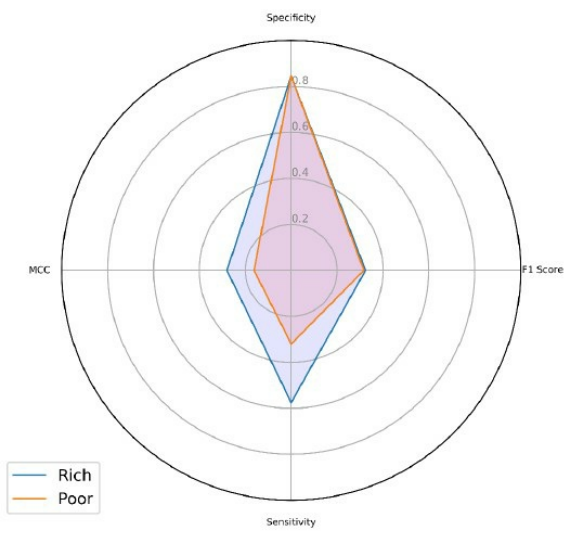

(B)

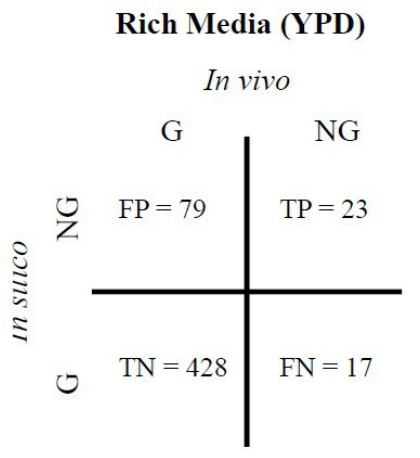

Minimal Media (YMMG)

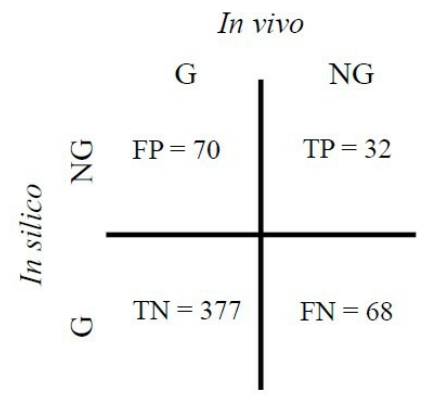

Figure 5

Single knockout analysis of viability in rich and minimal media. (A) Radar plot for comparative analysis of the model in rich and minimal media for classifying essential and non-essential genes over multiple statistical parameters i.e. F1 score, MCC, Precision, Recall and Accuracy. (B) Cross comparison between ground truth (Saccharomyces cerevisiae gene essentiality data) and the model predictions in rich and minimal media. NG and G stands for No Growth and Growth respectively for each in silico knockout. 
Fig 6
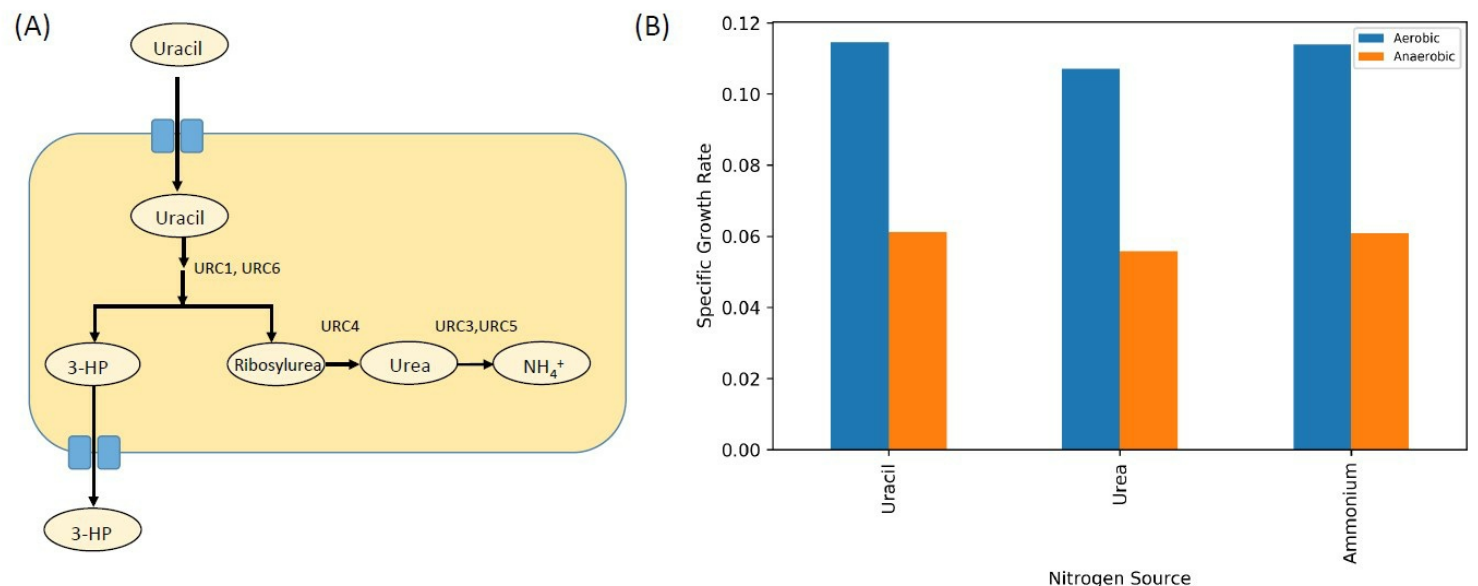

Figure 6

URC pathway for pyrimidine degradation in Lachancea kluyveri. (A) URC pathway in Lachancea kluyveri reported in literature. This enables the organism to assimilate Uracil and other pathway intermediates as it's sole nitrogen source. (B) The fitness in terms of specific growth rate for iPN730 simulated on uracil only or urea only or ammonium only as the sole nitrogen source.

\section{Supplementary Files}

This is a list of supplementary files associated with this preprint. Click to download.

Supporting_Information S1.xlsx

Lk_iPN730.xml 